University of Wollongong

Research Online

Faculty of Informatics - Papers (Archive)

Faculty of Engineering and Information

Sciences

1981

\title{
Higher dimensional orthogonal designs and applications
}

Joseph Hammer

Jennifer Seberry

University of Wollongong, jennie@uow.edu.au

Follow this and additional works at: https://ro.uow.edu.au/infopapers

Part of the Physical Sciences and Mathematics Commons

\section{Recommended Citation}

Hammer, Joseph and Seberry, Jennifer: Higher dimensional orthogonal designs and applications 1981. https://ro.uow.edu.au/infopapers/1005

Research Online is the open access institutional repository for the University of Wollongong. For further information contact the UOW Library: research-pubs@uow.edu.au 


\title{
Higher dimensional orthogonal designs and applications
}

\author{
Abstract \\ When $\mathrm{n} 2$ elements are given, they can be arranged in the form of a square; similarly, when $\mathrm{n} 8$ elements ( $\mathrm{g}$ \\ $\geq 3$ an integer) are given, they can be arranged in the form of a g-dimensional cube of side $n$ (in short, a g- \\ cube). The position of the elements can be indicated by $g$ suffixes. \\ Disciplines \\ Physical Sciences and Mathematics \\ Publication Details \\ Hammer, J and Seberry, J, Higher dimensional orthogonal designs and applications, IEEE Transactions on \\ Information Theory, Vol. IT-27(6), 1981, 772-779.
}




\title{
Higher Dimensional Orthogonal Designs and Applications
}

\author{
JOSEPH HAMMER AND JENNIFER R. SEBERRY
}

In memory of Professor G. Gáspár

\begin{abstract}
The concept of orthogonal design is extended to higher dimensions. A proper $g$-dimensional design $\left[d_{i j k} \ldots v\right]$ is defined as one in which all parallel $(g-1)$-dimensional layers, in any orientation parallel to a hyper plane, are uncorrelated. This is equivalent to the requirement that $d_{i j k \ldots v} \in\left\{0, \pm x_{1}, \cdots, \pm x_{t}\right\}$, where $x_{i}, \cdots, x_{t}$ are connuting variables, and that

$$
\sum_{p} \sum_{q} \sum_{r} \cdots \sum_{y} d_{p q r} \cdots y a d_{p q r} \cdots y b=\left(\sum_{i} s_{i} x_{i}^{2}\right)^{g-1} \delta a b,
$$

where $\left(s_{1}, \cdots, s_{t}\right)$ are integers giving the occurrences of $\pm x_{1}, \cdots, \pm x_{t}$ in each row and column (this is called the type $\left.\left(s_{1}, \cdots, s_{t}\right)^{z^{-1}}\right)$ and $(p q r \cdots y z)$ represents all pernutations of $(j j k \cdots v)$. This extends an idea of Pauj J. Shlichta, whose higher dimensional Hadamard matrices are special cases with $x_{1}, \cdots, x_{t} \in\{1,-1\},\left(s_{1}, \cdots, s_{t}\right)=(g)$, and $\left(\Sigma_{t} s_{i} x_{i}^{2}\right)$ $=\mathrm{g}$. Another special case is higher dimensional weighing matrices of type $(k)^{g}$, which have $x_{1}, \cdots, x_{t} \in\{0,1,-1\},\left(s_{1}, \cdots, s_{t}\right)=(k)$, and $\left(\sum_{t} s_{t} x_{i}^{2}\right)$ $=k$. Shlichta found proper $g$-dimensional Hadamard matrices of size $\left(2^{t}\right)^{g}$. Proper orthogonga designs of type $(1,1)^{3}$ and $(1, l, 1,1)^{3}$ are used to obtain higher dimensional orthogonal designs, Hadamard matrices, and weighing matrices. A possible approach to using higher dimensional weighing matrices and Hadanard matrices in codes is discussed, as welł as their connection with bigher dimensional orthogonal functions (Walsh, Haar, etc.).
\end{abstract}

\section{INTRODUCTION}

W HEN $n^{2}$ elements are given, they can be arranged in the form of a square; similarly, when $n^{g}$ elements ( $g \geq 3$ an integer) are given, they can be arranged in the form of a g-dimensional cube of side $n$ (in short, a $g$-cube). The position of the elements can be indicated by $g$ suffixes. Suppose there is a set of elements with $g$ suffixes $i j k \cdots v$ such as

$$
a_{i j k} \ldots,
$$

$n^{g}$ in number, arranged in $n^{g^{-2}}$ sets of two-dimensional square matrices in a space of $g$ dimensions and forming a $g$-dimensional cube of side $n$. The elements which have all the same suffixes, except $i$, lie in the same row (line); those which have all the same suffixes, with the exception of $i$ and/or $j$, lie in the same two-dimensional layer parallel to a coordinate axis (plane); and those which have only one in

Manuscript received January 9, 1979; revised Febnuary 2, 1981. This paper was presented at the Sixth Australian Combinatorics Conference, Armidale, N. S. W, Australia, August 1978.

J. Hammer is with the Pure Mathematics Department, University of Sydney, Sydney, New South Wales 2006, Australia.

J. R. Seberry is with the Applied Mathematics Department, University of Sydney, Sydney, New South Wales 2006, Australia. common lie in a $(g-1)$-dimensional layer. (For more particulars, consult Scott [13, pp. 110-119].)

Two-dimensional orthogonal designs [2] of type $\left(s_{1}, \cdots, s_{q}\right)$ may be defined as square orthogonal matrices with entries from $\left\{0, \pm x_{1}, \cdots, \pm x_{t}\right\}$, where $x_{1}, \cdots, x_{1}$ are commuting variables and $s_{j}$ is the number of times $\pm x_{j}$ occurs in each row and column-that is, in which all distinct rows and columns have scalar product zero. Hence the $n \times n\left(=n^{2}\right)$ matrix $\left[d_{i j}\right]$ is an orthogonal design of type $\left(s_{1}, \cdots, s_{t}\right)$ if it has entries from $\left\{0, \pm x_{1}, \cdots, \pm x_{t}\right\}$ and

$$
\sum_{i} d_{i a} d_{i b}=\sum_{j} d_{a j} d_{b j}=\sum_{k} s_{k} x_{k}^{2} \delta_{a b}
$$

Weighing matrices and Hadamard matrices (see [2]) are special cases in which the variables are from $\{0, \pm 1\}$ or $\{ \pm 1\}$, respectively and $\left(s_{1}, \cdots, s_{t}\right)$ is $(k)$ or $(n)$, respectively. Therefore, $\left[w_{i j}\right]$ is a weighing matrix $W(n, k)$ of side $n$ and weight $k$ if

$$
\sum_{i} w_{i a} w_{i b}=\sum_{j} w_{a j} w_{b j}=k \delta_{a b}
$$

and $\left[h_{i j}\right]$ is a Hadamard matrix if

$$
\sum_{i} h_{i a} h_{i b}=\sum_{j} h_{a j} h_{b j}=n \delta_{a b} .
$$

Aside from the trivial cases of $n=1$ and $n=2$, Hadamard matrices can exist only if $n$ is a multiple of four. The problem of finding the existence of at least one Hadamard matrix for all values of $n=4 t$ has been studied since 1892 [3], and the most powerful recent results [10] have depended entirely on the theory of orthogonal designs.

In recent years, Hadamard matrices have been used for a variety of practical applications in experimental design [12], error-correcting codes [12], signal processing [12], telephone networks [12], Walsh functions [4], [12], and high-speed multiplex spectrometry [I], [5].

The first person to use an orthogonal design to find Hadamard matrices was J. Williamson [14] in 1944. His work depended on using the orthogonal design of side 4 and type $(1,1,1,1)$

$$
\left[\begin{array}{rrrr}
x & y & u & v \\
-y & x & v & -u \\
-u & -v & x & y \\
-v & u & -y & x
\end{array}\right],
$$


and replacing the variables by four symmetric circulant $(1,-1)$ matrices $X, Y, U, V$ of side $t$ that satisfy

$$
X X^{T}+Y Y^{T}+U U^{T}+V V^{T}=4 t I_{t} .
$$

Subsequently, four symmetric circulant $(1,-1)$ matrices that satisfy (5), or just four $(1,-1)$ matrices that satisfy (5) and that in addition have $M N^{T}=N M^{T}$ for $N, M \in$ $\{X, Y, U, V\}$, have come to be known as Williamson matrices of side $t$. Williamson matrices of side $t$ can be used in (4) to obtain a Hadamard matrix of side $4 t$. We will use these matrices later to obtain higher dimensional Hadamard matrices.

Shlichta [8], [9] studied higher dimensional Hadamard matrices and gave elegant constructions for them in the case where the side is a power of two. He also introduced the concepts of proper and improper Hadamard matrices, which we discuss more fully in later sections. This paper generalizes the concept of orthogonal designs to higher dimensions, giving results about higher dimensional Hadamard matrices, weighing matrices, and other structures.

\section{First AND SECOND Definitions of ORTHOGONALITY}

There are several ways of defining orthogonality for higher dimensional matrices. One way is to say that a $\mathrm{g}$-dimensional matrix is orthogonal if its two-dimensional layers $M$ are orthogonal in all axis-normal directions - that is, if the inner product of their rows, considering them to be vectors, are pairwise zero, or equivalently if $M M^{T}=D_{m}$, a diagonal matrix of order $m$. We call this orthogonality by the first definition.

Another way to define orthogonality is to say that a $g$-dimensional matrix is orthogonal if its $(g-1)$ dimensional layers normal to one coordinate axis are mutually uncorrelated but are not in themselves orthogonal in any sense; moreover, the sets of $(g-1)$-dimensional layers normal to other axes are neither mutually uncorrelated nor orthogonal (i.e., in the sense of the present definition). We call this orthogonality by the second definition.

When we use the term "orthogonal" to describe a $g$ dimensional matrix without modifying the word orthogonal, we mean that its orthogonality lies between the extremes of the first and the second definitions of orthogonality.

These differing orthogonalities can be quantified by defining a $g$-dimensional matrix to be orthogonal of propriety $\left(d_{1}, \cdots, d_{g}\right)$ with $2 \leq d_{i} \leq g$, where $d_{i}$ indicates that in the $i$ th direction (i.e., the $i$ th coordinate), the $d_{i}-$ 1st, $d_{i}$ th,$d_{j}+$ Ist $\cdots,(g-1)$ th dimensional layers are mutually uncorrelated, but the $d_{i}-2$ nd dimensional layer is not. $d_{i}=\infty$ means that not even the $(g-1)$ st dimensional layers are orthogonal.

Thus, a g-dimensional matrix is said to be orthogonal by the first definition if it has propriety $(2,2, \cdots, 2)$ and orthogonal by the second definition if it has propriety $(\infty, \infty, \cdots, \infty, g, \infty, \cdots, \infty)$. (Compare this classification of orthogonality with that of Shlichta $[8, \S \S 2,5]$ for
Hadamard matrices: Shlichta's proper Hadamard matrices have propriety $(2,2, \cdots, 2)$.) Shlichta's definition of orthogonality requires that for propriety $\left(d_{1}, d_{2}, \cdots, d_{n}\right), 2 \leq d_{i}$ $\leq \mathrm{g}, i=1, \cdots, n$.

We have been motivated to consider matrices of propriety $(\infty, \infty, \cdots, \infty)$ by the highly structured Paley cube found in the next section and higher dimensional Walsh functions. Let a matrix $A$ be denoted by

$$
A=\left[\begin{array}{c}
a^{1} \\
a^{2} \\
\vdots \\
a^{n}
\end{array}\right]
$$

where $a^{i}=\left(a_{1}^{i}, a_{2}^{i}, \cdots, a_{n}^{i}\right), i=1, \cdots, n$. Similarly, let

$$
B=\left[\begin{array}{c}
b^{1} \\
\vdots \\
b^{n}
\end{array}\right] .
$$

By the inner product of $A$ and $B$ will be meant

$$
A \cdot B=a^{1} \cdot b^{1}+a^{2} \cdot b^{2}+\cdots+a^{n} \cdot b^{n} \text {. }
$$

Alternatively, $A \cdot B$ can be written as the sum of the diagonal elements of $A B^{T}$, which is in fact $\operatorname{tr}\left(A B^{T}\right)$.

From the rows of a given orthogonal square matrix $H$, we construct layers of a three-cube orthogonal by the second definition in the following way.

Let $H$ be given by

$$
\boldsymbol{H}=\left[\begin{array}{c}
\boldsymbol{h}^{1} \\
\boldsymbol{h}^{2} \\
\vdots \\
\boldsymbol{h}^{n}
\end{array}\right]=\left[\begin{array}{cccc}
h_{1}^{1}, & h_{2}^{\mathrm{j}}, & \cdots, & h_{n}^{1} \\
h_{1}^{2}, & h_{2}^{2}, & \cdots, & h_{n}^{2} \\
\vdots & \vdots & & \vdots \\
h_{1}^{n}, & h_{2}^{n}, & \cdots, & h_{n}^{n}
\end{array}\right]
$$

where $\boldsymbol{h}^{p} \cdot \boldsymbol{h}^{q}=0, p, q=1, \cdots, n, p \neq q$. Orthogonal layers $H(i, j)$ are obtained by taking the tensor products of the vectors $\boldsymbol{h}^{i}, \boldsymbol{h}^{j}$ :

$$
\begin{aligned}
H(i, j) & =\boldsymbol{h}^{i} \otimes\left(\boldsymbol{h}^{j}\right)^{T} \\
& =\left[\begin{array}{cccc}
h_{1}^{i} h_{1}^{j}, & h_{1}^{i} h_{2}^{j}, & \ldots, & h_{1}^{i} h_{n}^{j} \\
h_{2}^{i} h_{1}^{j}, & h_{2}^{i} h_{2}^{j}, & \cdots, & h_{2}^{i} h_{n}^{j} \\
\vdots & \vdots & & \vdots \\
h_{n}^{i} h_{1}^{j}, & h_{n}^{i} h_{2}^{j}, & \cdots, & h_{n}^{i} h_{n}^{j}
\end{array}\right]=\left[\begin{array}{c}
h_{1}^{i} \boldsymbol{h}^{j} \\
h_{2}^{i} \boldsymbol{h}^{j} \\
\vdots \\
h_{n}^{i} \boldsymbol{h}^{j}
\end{array}\right] .
\end{aligned}
$$

It is easy to check that $H(i, j) \cdot H(k, l)=0$, for all $i, j, k, l=1, \cdots, n, j \neq l$. For

$$
\begin{aligned}
H(i, j) \cdot \boldsymbol{H}(k, l) & =\left(h_{1}^{i} \boldsymbol{h}^{j}\right) \cdot\left(h_{1}^{k} h^{l}\right)+\left(h_{2}^{i} h^{j}\right) \cdot\left(h_{2}^{k} h^{l}\right) \\
& +\cdots+\left(h_{n}^{i} h^{j}\right) \cdot\left(h_{n}^{k} h^{l}\right) \\
= & \left(h_{1}^{i} h_{1}^{k}+h_{2}^{i} h_{2}^{k}+\cdots+h_{n}^{i} h_{n}^{k}\right)\left(\boldsymbol{h}^{j} \cdot \boldsymbol{h}^{l}\right) \\
= & 0
\end{aligned}
$$

as $\boldsymbol{h}^{j} \cdot \boldsymbol{h}^{l}=0$, for all $l, j=1, \cdots, n, l \neq j$.

We construct fourth and higher dimensional orthogonal matrices that arz orthogonal by the second definition in an analogous way. Denote a $g$-dimensional matrix by $[M]_{g}$ 
and the $(g-1)$-dimensional layers by $[M]_{g-1}$. Now the inner product of two $g$-cubes $[A]_{g}$ and $[B]_{g}$ is the sum of the inner products of their respective $(g-1)$-layers parallel to the $i$ th coordinate plane $i=1, \cdots, g$, e.g.,

$$
[A]_{g} \cdot[B]_{g}=\sum_{j=1}^{n}\left[A_{j}^{i}\right]_{g-1} \cdot\left[B_{j}^{i}\right]_{g-1},
$$

where $A_{j}^{i}$ denotes the $j$ th layer parallel to the $i$ th coordinate plane. We say that $[\mathrm{H}]_{g}$ is orthogonal if the inner product of the $(g-1)$-dimensional layer matrices parallel to a coordinate hyperplane is pairwise equal to zero. Again we can construct such $(g-1)$-dimensional layers by taking the tensor products of the layers of a $(g-1)$-dimensional orthogonal cube.

Remark: This second defintion of orthogonality of higher dimensional matrices is quite in agreement with the orthogonality of the two-dimensional matrices from the point of view of dimensions. In a two-dimensional matrix $M$, the layers are the rows (or columns) of $M$ that can be assumed as one-dimensional matrices, i.e., one dimension lower than the dimension of $M$, so that in this case, too, orthogonality can be defined as the inner product of the parallel layer matrices pair-wise equal to zero.

Consider the $g$-cube $[H]_{g}$ of side $h$, and denote $1-$, $2-, \cdots,(g-1)$-dimensional layer matrices by $\left[H^{j}\right]_{1}$, $\left[\mathrm{H}^{i}\right]_{2}, \cdots,\left[H^{i}\right]_{g-l}$, where $i$ represents the layers embedded in the subspaces parallel to the $i$ th coordinate hyperplane. If for all pairs of distinct one-dimensional layers $\left[H^{j}\right]_{1}$ and $\left[H^{i}\right]_{1}^{\prime}$ parallel to the $i$ th coordinate hyperplane we have $\left[H^{i}\right]_{1} \cdot\left[H^{i}\right]_{1}^{i}=0$, then for all distinct pairs of twodimensional layers $\left[H^{i}\right]_{2}$ and $\left[H^{i}\right]_{2}^{\prime} \cdot\left[H^{i}\right]_{2}^{\prime}=0$, and so on, i.e.,

$$
\begin{aligned}
{\left[H^{i}\right]_{1} \cdot\left[H^{i}\right]_{1}^{\prime} } & =0 \Rightarrow\left[H^{i}\right]_{2} \cdot\left[H^{i}\right]_{2}^{\prime}=0 \\
& \Rightarrow \cdots \Rightarrow\left[H^{i}\right]_{g-1} \cdot\left[H^{i}\right]_{g-1}^{\prime}=0 .
\end{aligned}
$$

It can be observed that $\left[H^{i}\right]_{1} \cdot\left[H^{i}\right]_{1}^{i}=0$ is the first definition of orthogonality, provided that $i$ takes up all values from 1 to $g$, and

$$
\left[H^{i}\right]_{g-1} \cdot\left[H^{i}\right]_{g-1}^{\prime}=0
$$

is the second definition of orthogonality. In other words, orthogonality by the first definition implies orthogonality by the second definition. A similar relationship was pointed out by Shlichta [9]. Between the first and second definitions we can have a large variety of orthogonalities according to the dimension of the layers, which can vary from 1 to $g-1$, and the numbers of coordinate hyperplanes to which the layers can be parallel, which can go from 1 to $g$. The two extreme cases are the first and second definitions.

\section{EXAMPLES AND REMARKS}

In fact, if $A$ and $B$ are two $g$-cubes orthogonal of proprieties $\left(a_{1}, \cdots, a_{g}\right)$ and $\left(b_{1}, \cdots, b_{g}\right)$ and sides $a$ and $b$, respectively, then the Kronecker product $A \otimes B$ of $A$ and $B$ obtained by substituting $A b_{i j} \ldots$ for each entry $b_{i j} \ldots v$ of $B$ is a $g$-cube of side $a b$ orthogonal of propriety $\left(c_{1}, \cdots, c_{g}\right)$, where $c_{i}=\max \left(a_{i}, b_{i}\right), i=1, \cdots, g$.

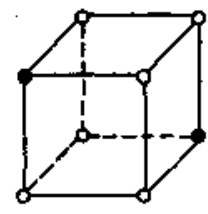

(a)

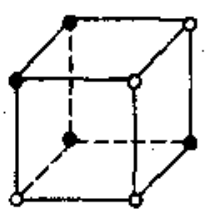

(b)
Fig. 1. $2^{3}$ Hadamard matrix of propriety $(2,2,2)$, and three-dimensional matrix of propriety $(2,2,3)$. (a) Proper. (b) Improper.

A set $\mathfrak{E}=\left\{A_{1}, A_{2}, \cdots, A_{n}\right\}$ of $g$-cubes orthogonal of proprieties $\left(a_{i 1}, a_{i 2}, \cdots, a_{i g}\right)$, where $a_{i j} \leq \max$ $\left\{a_{i 1}, a_{i 2}, \cdots, a_{i n}\right\}$ for $i=1, \cdots, n$ generates a monoid under the operation $\otimes$, i.e., it satisfies the following properties:

1) $A_{i} \otimes A_{j}$ is orthogonal;

2) $A_{i} \otimes\left(A_{j} \otimes A_{k}\right)=\left(A_{i} \otimes A_{j}\right) \otimes A_{k}$; and

3) $I_{1 \times 1} \in \mathbb{Q}$ is the unit element.

Since $A_{j} \otimes A_{j}$ is equivalent to $A_{j} \otimes A_{i}$ by an appropriate permutation of rows and columns, we could say that the monoid is "combinatorially commutative."

Example 1: The front face and back face of Fig. 1(b) are, as vectors (with $\bar{l}=-1$ ),

$$
(\overline{1}, 1,1,1) \quad(\overline{1}, 1, \overline{1}, 1) .
$$

These are orthogonal, and so these faces (or parallel twodimensional layers) are said to be orthogonal. The similar vectors for Fig. 1(a) are

$$
(\overline{1}, 1,1,1) \quad(1,1,1, \overline{1}) \text {. }
$$

Now all the two-dimensional layers are orthogonal for both Figs. 1(a) and 1(b), but in Fig. l(a) every face is a Hadamard matrix and so is orthogonal of propriety $(2,2,2)$, while Fig. $1(b)$ is orthogonal of propriety $(2,2,3)$.

Example 2 (the Paley cube): Let $q \equiv 3(\bmod 4)$ be a prime power and $z_{0}=0, z_{1}=1, \cdots, z_{q-1}$ be the elements of $\mathrm{GF}(q)$, the Galois field. We define

$$
p_{i j} \ldots,= \begin{cases}+1, & \text { if any of the subscripts is } q, \\ x\left(z_{i}+z_{j}+\cdots+z_{r}\right), & \text { otherwise, }\end{cases}
$$

where each subscript runs from zero to $q, P=\left[p_{i j} \ldots r\right)$ is a $(q+1)$-dimensional Paley cube, and

$$
\begin{aligned}
& \chi(0)=-1 \\
& \chi(z)= \begin{cases}+1, & \text { if } z \text { is a square in } \operatorname{GF}(q), \\
-1, & \text { otherwise. }\end{cases}
\end{aligned}
$$

By the same reasoning as before, and using the twodimensional properties of this matrix (see [2]), we see that each two-dimensional face of the Paley cube except the one that contains all ones is a Hadamard matrix. (See Fig. 2.)

So then, when $q \equiv 3(\bmod 4)$ is a prime power, there is an almost Hadamard $(q+1)$-dimensional cube, called the Paley cube, of side $q+1$, that has one two-dimensional layer in each direction all ones and every other face a 


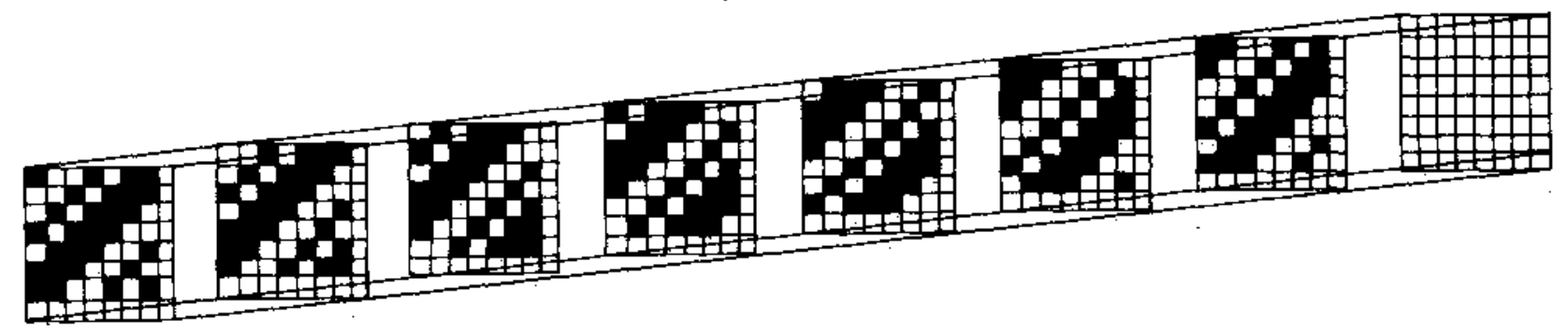

Fig. 2. Paley cube of dimension 3, 1 Minus one. $\square$ Plus one.

Hadamard matrix. The Paley cube has propriety $(\infty, \infty, \cdots, \infty)$, but if the two-dimensional layer of all ones is removed in one direction, the remaining $g$-dimensional matrix (note it is no longer a cube) has all two-dimensional layers in that direction orthogonal.

Nevertheless, the Paley cube, while not itself orthogonal, promises to be highly useful in error-correcting codes, because of the great number of orthogonal two-dimensional matrices within the cube.

\section{ORThOGONAL DESIGNS}

A proper g-cube orthogonal design $D=\left[d_{i j k} \ldots.\right]$ of side $d$ and type $\left(s_{1}, s_{2}, \cdots, s_{t}\right)^{g}$ is one in which all parallel two-dimensional layers, in any orientation parallel to a plane, are uncorrelated. This is equivalent to the requirement that $d_{i j k} \cdots_{v} \in\left\{0, \pm x_{1}, \cdots, \pm x_{t}\right\}$, where $x_{1}, \cdots, x_{i}$ are commuting variables, and that

$\sum_{p} \sum_{q} \sum_{r} \cdots \sum_{y} d_{p q r} \cdots y a d p d_{p q r} \cdots y b=\left(\sum_{t} s_{i} x_{i}^{2}\right)^{g \cdots 1} \delta_{a b}$,

where $\left(s_{1}, \cdots, s_{t}\right)$ are integers giving the occurrences of $\pm x_{1}, \cdots, \pm x_{t}$ in each row and column (this is called the type $\left.\left(s_{1}, \cdots, s_{t}\right)^{g}\right)$, and $(p q r \cdots y z)$ represents all permutations of $(i j k \cdots v)$. This is by the first definition (see Section II), i.e., it is of propriety $(2,2, \cdots, 2)$. In a similar fashion to Section III it is possible to define orthogonal designs by the second definition or by any other propriety of orthogonality.

Higher dimensional orthogonal designs may be constructed by noting that if $A$ is a $g$-cube orthogonal design of side $a$ and type $\left(s_{1}, s_{2}, \cdots, s_{t}\right)^{g}$, and $H$ is a g-dimensional Hadamard matrix of side $h$, then $H \otimes A$ is a $g$-cube orthogonal design of side $h a$ and of type $\left(h s_{1}, h s_{2}, \cdots, h s_{q}\right)^{g}$. The propriety depends on the propriety of the matrices used.

Example: Call Fig. 1(a) $A_{2}$ and Fig. 3(a) $A_{1}$. Then $A_{1} \otimes A_{2}$ is given in Fig. 4.

\section{Higher Dimensional Orthogonal Designs OF $\operatorname{TrPES}(1,1)^{3}$ AND $(1,1,1)^{3}$}

These orthogonal designs are illustrated in Figs. 3 and 5. It can be seen in Fig. 5 that the orthogonal design of type $(1,1,1,1)^{3}$ and side 4 can be constructed from basic 3 -cubes of side two and type $(1,1)^{3}$ by suitable orientation. These 3-cubes are crucial for our construction of higher dimensional weighing matrices and Hadamard matrices.

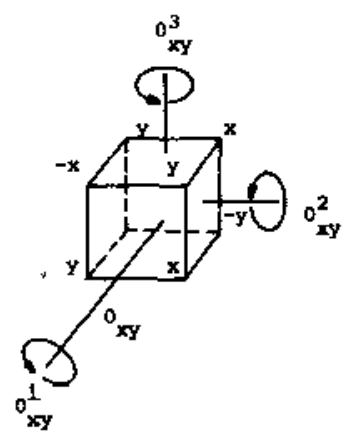

(a)
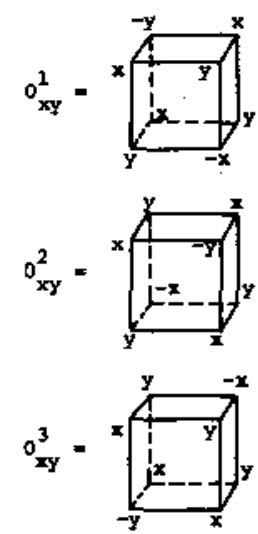

(b)
Fig. 3. Orthogonal design of side 2 and type $(1,1)^{3}$. (a) Basic $(1,1)^{3}$ cube. (b) $(1,1)^{3}$ designs obtained by rotation.

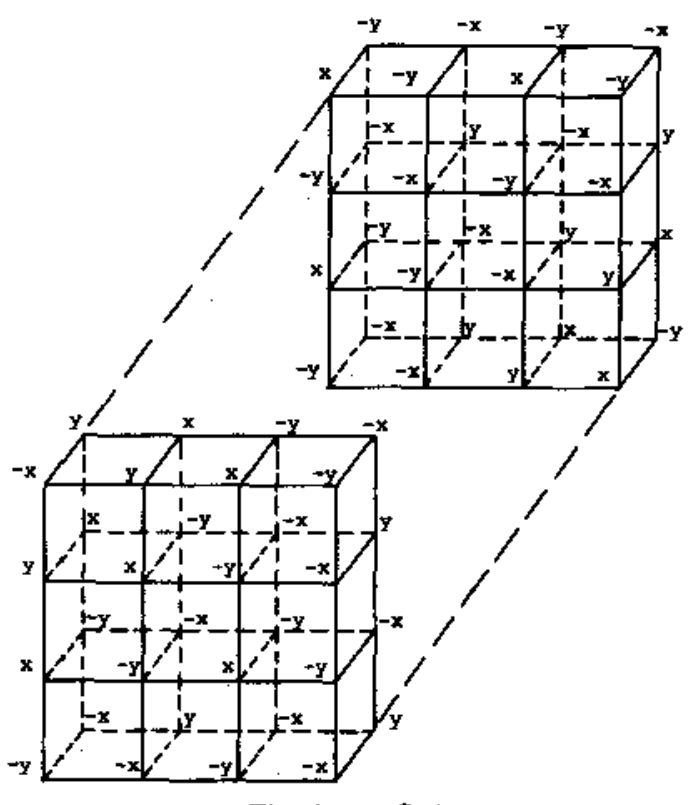

Fig. 4. $A_{1} \otimes A_{2}$.

\section{VI. $t$-DIMENSIONAL Matrices from SUITABLE MATRICES}

Let $z_{1}, z_{2}, \cdots, z_{n}$ be the elements of an abelian group $G$ of order $n$. A type 2 or $l$ matrix (see [2]) $A=\left[a_{i j}\right]$ is one defined by

$$
a_{i j}=\alpha\left(z_{i} \pm z_{j}\right)
$$




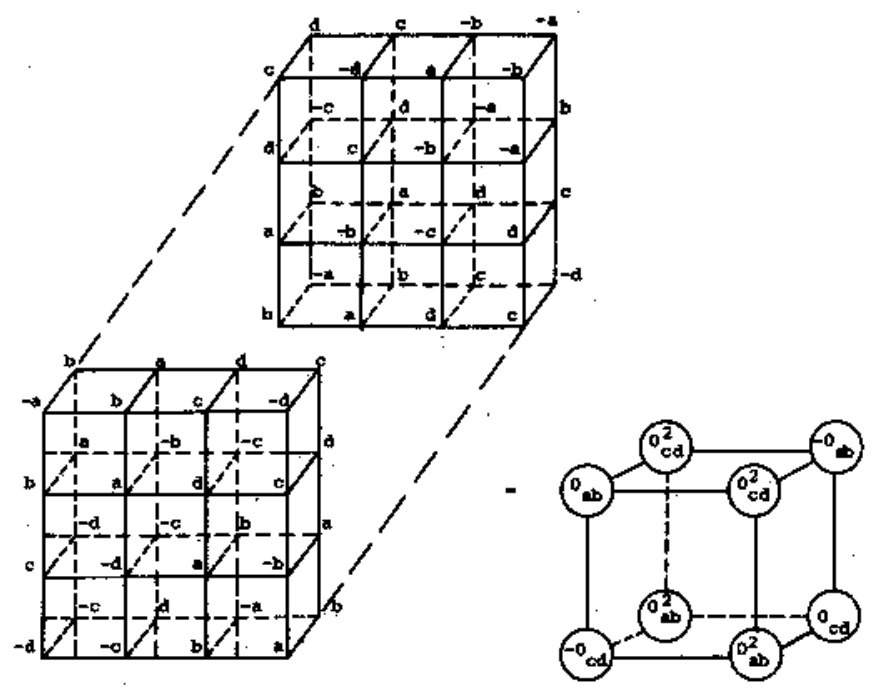

Fig. 5. Orthogonal design of side 4 and type $(1,1,1,1)^{3}$.

where $\alpha$ is a map into a commutative ring. A circulant matrix of order $n$ is a special case in that $G=Z_{n}$ (the cyclic group of order $t$ ), with $z_{1}=1, z_{2}=2, \cdots, z_{n}=n$, and so

$$
\begin{aligned}
a_{i j} & =\alpha(i \pm j) \\
& = \begin{cases}\alpha(i+j), & \text { for type } 2 \text { matrices, } \\
\alpha(i-j), & \text { for type } 1 \text { matrices. }\end{cases}
\end{aligned}
$$

A set of $m$ matrices $X_{1}, X_{2}, \cdots, X_{m}$ of order $n$ with $X_{k}=\left[x_{i j}^{k}\right]$ is called $m$-suitable matrices if

$$
\begin{aligned}
\sum_{k=1}^{m} \sum_{i=1}^{n}\left[x_{a i}^{k}\right]\left[x_{b i}^{k}\right] & =f \delta_{a b}, \\
\sum_{i=1}^{n}\left[x_{a i}^{k}\right]\left[x_{b i}^{I}\right] & =\sum_{i=1}^{n}\left[x_{a i}^{l}\right]\left[x_{b i}^{k}\right],
\end{aligned}
$$

where $f$ is a constant or constant function. Williamson matrices of order $t$, which we mentioned earlier, are 4suitable matrices with entries \pm 1 and $f=4 t$.

In [2] it is proven that type 1 matrices commute, and so symmetric type 1 matrices always satisfy (10). Furthermore, if $A$ is a type 1 matrix, there exists a permutation matrix $R$ such that $A R$ is a type 2 matrix and

$$
A A^{T}=(A R)(A R)^{T} \text {. }
$$

This is used in [2] to show that when the elements of the Abelian group are ordered so that the first rows of type 2 matrices $y_{j}, \cdots, y_{j n}$ satisfy $y_{j, 2+i}=y_{j, n-1}$, then such type 2 matrices commute and so satisfy (10).

Suppose $X_{k}=\left[x_{i j}^{k}\right], k=1, \cdots, m$ are $m$-suitable type 2 matrices of order $t$ defined, similarly to (8), by

$$
x_{i j}^{k}=\psi_{k}\left(z_{i}+z_{j}\right)
$$

Then from (9) and (10) we have

$$
\begin{array}{r}
\sum_{k=1}^{m} \sum_{i=1}^{t} \psi_{k}\left(z_{a}+z_{i}\right) \psi_{k}\left(z_{b}+z_{i}\right) \\
=f \delta_{a b}=\sum_{k=1}^{m} \sum_{g \in G} \psi_{k}\left(z_{a}+g\right) \psi_{k}\left(z_{b}+g\right), \\
\sum_{i=1}^{t} \psi_{k}\left(z_{a}+z_{i}\right) \psi_{l}\left(z_{b}+z_{i}\right)=\sum_{i=1}^{t} \psi_{l}\left(z_{a}+z_{i}\right) \psi_{k}\left(z_{b}+z_{i}\right) .
\end{array}
$$

We define the elements of the $t$-dimensional cube for each $k$ by

$$
x_{i j p}^{k} \ldots t=\psi_{k}\left(z_{i}+z_{j}+z_{p}+\cdots+z_{s}\right) \text {. }
$$

To consider the inner product properties of the twodimensional faces of this cube, we let the $q$ th coordinate take two values $a$ and $b$, and the $r$ th coordinate run from 1 to $t$, all other coordinates being constant. Then, with $y=$ sum of all $z_{i}, \cdots, z_{t}$ except the $q$ th and $r$ th coordinates, $z_{q}$ and $z_{r}$,

$$
\begin{aligned}
& \sum_{m=1}^{t} x_{i j p}^{k} \cdots a \cdots m \cdots, x_{i j p}^{k} \cdots b \cdots m \cdots t \\
& =\sum_{m=1}^{t} \psi_{k}\left(y+z_{a}+z_{m}\right) \psi_{k}\left(y+z_{b}+z_{m}\right) \\
& =\sum_{g \in G} \psi_{k}\left(y+z_{a}+g\right) \psi_{k}\left(y+z_{b}+g\right) \\
& =\sum_{h \in G} \psi_{k}\left(z_{a}+h\right) \psi_{k}\left(z_{b}+h\right),
\end{aligned}
$$

since $m=1, \cdots, t$ means that $z_{m}$ runs through $G$, and $g$ running through $G$ means that $y+g$ runs through $g$.

To find the inner product of the rows of the corresponding two-dimensional layers in different $t$-dimensional matrices, we let the $r$ th coordinate sum from one to $t$, the $q$ th coordinate take two values ( $a$ and $b$ ), and all other coordinates remain constant. Write $y$ equal to the sum of all $z_{1}, \cdots, z_{t}$ except the $q$ th and $r$ th coordinates. Then,

$$
\begin{aligned}
& \sum_{m=1}^{t} x_{i j p}^{k} \cdots a \cdots m \ldots t x_{i j p}^{l} \cdots b \cdots m \cdots t \\
& =\sum_{m=1}^{t} \psi_{k}\left(y+z_{a}+z_{m}\right) \psi_{l}\left(y+z_{b}+z_{m}\right) \\
& =\sum_{i=1}^{t} \psi_{k}\left(z_{a}+z_{i}\right) \psi_{i}\left(z_{b}+z_{i}\right) \\
& =\sum_{i=1}^{t} \psi_{i}\left(z_{a}+z_{i}\right) \psi_{k}\left(z_{b}+z_{i}\right) \quad \text { using (12) } \\
& =\sum_{m=1}^{t} x_{i j p}^{t} \ldots b \cdots m \ldots x_{i j p}^{k} \ldots a \cdots m \cdots t .
\end{aligned}
$$


Higher dimensional Hadamard matrices may be constructed by replacing the variables of a $g$-cube orthogonal design of type $(1,1, \cdots, 1)^{g}$, where there are $m$ ones, by the $m$-suitable matrices.

Combining (11) and (14), we see that if the rows of $m$-suitable matrices are, on addition, orthogonal, the rows of the $t$-dimensional matrices formed from these matrices, in any direction parallel to the axis, will, on addition, also be orthogonal. The contribution from the different $m$ suitable matrices is canceled out in the orthogonal design by use of (15).

\section{ApPlying Orthogonal Designs to Form Higher dimensional WEIGHING Matrices}

In the orthogonal design of side 2 and type $(1,1)^{3}$ (see Fig. 3), which we call $\left[d_{i j k}\right]$, we have (with $\bar{l}=-1$ )

$$
\dot{x}=-d_{000}=d_{10 \overline{1}}=d_{110}=d_{01 \overline{1}}
$$

and

$$
y=d_{001}=d_{0 t 0}=d_{100}=-d_{11 \overline{1}} .
$$

Suppose that $\left[x_{i j}^{1} \ldots t\right]$ and $\left[x_{i j}^{2} \ldots t\right]$ are $t$-dimensional matrices formed from 2 -suitable matrices. Then we form a three-dimensional weighing matrix of propriety $(2,2,2)$ and side $2 t\left[w_{i j} \ldots t\right]$ (i.e., each subscript $i, j, \cdots, t$ can assume any value $1,2, \cdots, 2 t$ ) by replacing each $\pm x$ in the 3 -cube orthogonal design of order two and type $(1,1)^{3}$ by $\pm\left[x_{i j}^{1} \ldots,\right]$ and each $\pm y$ by $\left[x_{i j}^{2} \ldots,\right]$. This is illustrated for the 2-suitable matrices

$$
\left[\begin{array}{lll}
\overline{1} & 1 & 1 \\
1 & 1 & 1 \\
1 & 1 & 1
\end{array}\right] \quad\left[\begin{array}{lll}
0 & 1 & 1 \\
1 & 0 & 1 \\
1 & 1 & 0
\end{array}\right]
$$

in Fig. 6, where a 3-cube weighing matrix of side 6 and propriety $(2,2,2)$ with five nonzero entries in each row and column is illustrated (i.e., type $\left.(5)^{3}\right)$.

It is known that 2-suitable matrices exist for orders $\frac{1}{2}(q+1)$, when $q \equiv 1(\bmod 4)$ is a prime power (see [2]), that are circulant and symmetric. This ensures that the inner products from rows of $\left[x^{1}\right]$ and $\left[x^{2}\right]$ will cancel out.

Hence, when $q \equiv 1(\bmod 4)$ is a prime power, there exist three-dimensional weighing matrices of side $q+1$ and propriety $(2,2,2)$ with $q$ nonzero elements in each row and column (i.e., of type $\left.(q)^{3}\right)$.

\section{APPlying OrThogonal Designs to Form Higher Dimensional Hadamard Matrices}

Williamson matrices of order $t$ (or 4-suitable matrices in our present terminology) exist for the following orders (see [15], [7]):

$$
\begin{array}{lll}
\text { W I } & & \text { where } t \in(1,3,5, \cdots, 29,37,43\} ; \\
\text { W II } & \frac{1}{2}(p+1) & p \equiv 1(\bmod 4) \text { a prime power; } \\
\text { W III } & 3^{c}, 7.3^{c-1} & c \text { is a natural number; }
\end{array}
$$

\begin{tabular}{|c|c|c|}
\hline W IV & $\frac{1}{2} p^{r}(p+1)$ & $\begin{array}{l}p \equiv 1(\bmod 4) \text { a prime power, } r \text { a } \\
\text { natural number; }\end{array}$ \\
\hline W V & $s(4 s-1)$ & $\begin{array}{l}s \text { is the order of a good matrix } \\
\text { (see [11]) for definition); }\end{array}$ \\
\hline W VI & $s(4 s+3)$ & $\begin{array}{l}s \text { is the order of a good matrix, } \\
4 s+4 \text { is the order of a symmetric }\end{array}$ \\
\hline W VII & so & $\begin{array}{l}s \text { is the order of a good matrix, } v \\
\text { is the order of an Abelian group } \\
G \text { on which are defined a }(v, k, \lambda) \\
\text { and a }(v,(v-1) / 2,(v-s) / 4)) \\
\text { difference set } v-4(k-\lambda)= \\
4 s-1 \text {. }\end{array}$ \\
\hline
\end{tabular}

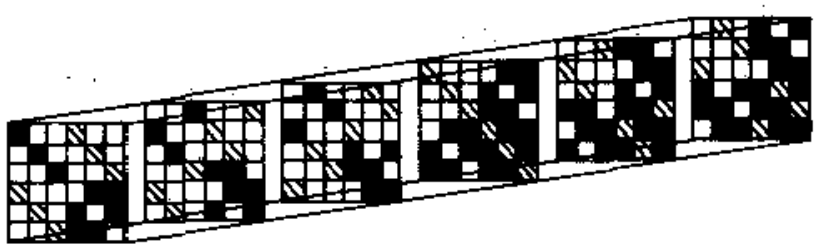

Fig. 6. Weighing matrix of side 6 and dimension $3\left(=\operatorname{order} 6^{3}\right)$, with propriety $(2,2,2)$., $\square$ Zero. $\otimes$ Plus one. $\square$ Minus one.

This means that the only odd $t<100$ for which Williamson matrices are not yet known are $35,39,47,53,59,65$, $67,71,73,77,83$, and 89 .

The Williamson matrices are used to form $t$-dimensional cubes $\left[x_{i j}^{1} \ldots p\right],\left[x_{i j}^{2} \ldots p\right],\left[x_{i j}^{3} \ldots p\right]$, and $\left[x_{i j}^{4} \ldots p\right]$, which are used to replace $a, b, c$, and $d$ of the orthogonal design of side 4 and type $(1,1,1,1)^{3}$ (see Fig. 5.) Because of the properties of these matrices, each of the faces parallel to the axes will be a Hadamard matrix of propriety $(2,2,2)$.

Thus, when $t$ is the side of 4 Williamson matrices, there is a three-dimensional Hadamard matrix of side $4 t$ and propriety $(2,2,2)$. (That is, there is a $(4 t)^{3}$ Hadamard matrix of propriety $(2,2,2)$ ).

\section{Application to Multivariable Discrete ORTHOGONAL FUNCTIONS}

Higher dimensional orthogonal matrices (according to the second definition) can be applied usefully to represent multivariable discrete orthogonal functions. (For such functions, consult $[4$, pp. $36-44]$.)

For instance, the rows of the Walsh-Hadamard matrix (where the minus sign stands for -1 )

$$
[W]_{2}=\left[\begin{array}{cccc}
1 & 1 & 1 & 1 \\
- & - & 1 & 1 \\
- & 1 & 1 & - \\
1 & - & 1 & -
\end{array}\right]
$$

represent four elements of a Walsh function of one dimension.

By the procedure described in Section III, we can construct an orthogonal matrix of three dimensions. Its layers represent the elements of a Walsh function of two dimen- 
sions. Four elements of a Walsh 3-cube are

$$
\begin{gathered}
{\left[\begin{array}{llll}
1 & 1 & 1 & 1 \\
1 & 1 & 1 & 1 \\
1 & 1 & 1 & 1 \\
1 & 1 & 1 & 1
\end{array}\right], \quad\left[\begin{array}{cccc}
- & - & 1 & 1 \\
- & - & 1 & 1 \\
- & - & 1 & 1 \\
- & - & 1 & 1
\end{array}\right],} \\
{\left[\begin{array}{cccc}
1 & 1 & 1 & 1 \\
1 & 1 & 1 & 1 \\
- & - & - & -
\end{array}\right], \quad\left[\begin{array}{llll}
- & - & 1 & 1 \\
- & - & 1 & 1 \\
1 & 1 & - & - \\
1 & 1 & - & -
\end{array}\right] .}
\end{gathered}
$$

This cube has propriety $(3, \infty, \infty)$; i.e., it is orthogonal by the second definition.

Similarly, from certain two-dimensional orthogonal matrices with entries $0,1,-1$ that represent elements of one-dimensional Haar functions, we can construct orthogonal two-dimensional Haar functions.

\section{Application to Discrete Orthogonal TRANSFORMATIONS}

It is well-known (Ahmed and Rao [1], Harwit and Sloane [5]) that a discrete Walsh-Hadamard transformation in one variable can be expressed by the matrix equation

$$
\boldsymbol{a}=\boldsymbol{W},
$$

where

$$
\boldsymbol{a}=\left[\begin{array}{c}
a_{1} \\
a_{2} \\
\vdots \\
a_{N}
\end{array}\right] \quad \boldsymbol{x}=\left[\begin{array}{c}
x_{1} \\
x_{2} \\
\vdots \\
x_{N}
\end{array}\right]
$$

are the output and input vectors of $N$ sampling points. $W$ is a Hadamard-Walsh symmetric matrix with entries 1 or -1 of order $N=2^{p}, p$ being a positive integer such that the rows of $W$ are in a special ordering. Similarly, a $\mathrm{Haar}$ transformation has an orthogonal matrix with entries $0,1,-1$ in a special ordering. There are several other important discrete orthogonal transformations that can be found in the references.

The inverse transformation can be written from (16) as

$$
\boldsymbol{x}=W^{-1} \boldsymbol{a},
$$

where $W^{-1}$ is the inverse of $W$.

Now a discrete Hadamard transform of two variables can be written by the following matrix equation:

$$
[A]_{2}=[W]_{3}[X]_{2} \text {, }
$$

where

$$
\begin{aligned}
& {[A]_{2}=\left[a^{1}, a^{2}, \cdots, a^{N}\right] \text { and }} \\
& {[X]_{2}=\left[x^{1}, x^{2}, \cdots, x^{N}\right],}
\end{aligned}
$$

and $[\mathrm{W}]_{3}$ is a Walsh 3-cube (or a Haar 3-cube, or some other orthogonal cube) of layers $\left[W_{i}^{j}\right]_{2}, i=1, \cdots, N$ (i.e., orthogonal by the second definition). By the product $[W]_{3}[X]_{2}$ is meant

$$
[W]_{3}[X]_{2}=\left(\left[W_{1}^{j}\right]_{2} \mathbf{x}^{1},\left[W_{2}^{J}\right]_{2} x^{2}, \cdots,\left[W_{N}^{j}\right]_{2} x^{N}\right) \text {. }
$$

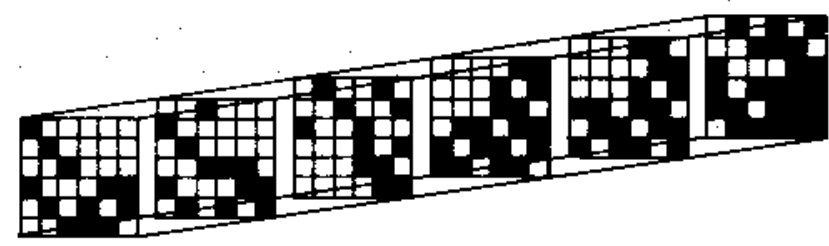

Fig. 7. Three-dimensional matrix of propriety $(3,3, \infty)$.

From this, (18) can be written as $a^{1}=\left[W^{j}\right]_{2} x^{1}, a^{2}=$ $\left[W_{2}\right]_{2} x^{2}, \cdots, a^{N}=\left[W_{N}^{j}\right]_{2} x^{N}$; i.e., (18) represents $N^{2}$ scalar equations.

The inverse of $(18)$ is written as

$$
[X]_{2}=\left([\dot{W}]_{3}\right)^{-1}[A]_{2},
$$

where, by definition, $\left([W]_{3}\right)^{-1}$ is the inverse of the layers of $[W]_{3}$.

The extension of (18) for a discrete $(g-1)$-variable transformation is

$$
[A]_{g-1}=[W]_{g}[X]_{g-1},
$$

where the input and output matrices are one dimension less than the transform cube $[W]_{g}$. The product of $[W]_{g}[X]_{g-1}$ is, by definition, the product of layers of $[W]_{g}$ by the respective layers of $[X]_{g-1}$.

\section{Application to Error Correcting Codes}

It is not necessary to have matrices of propriety $(2,2, \cdots, 2)$ to have an error correcting code. For example, Fig. 6 is an orthogonal matrix of side 6 and dimension 3 . The side faces of the 3-cube of Fig. 7 are never orthogonal: the matrix is of propriety $(3,3, \infty)$.

In this case we can either have two-dimensional codewords of distance 18 or regard each face as a codeword of length 36 and distance 18 that has been folded into 6 strings (vectors) of length 6 .

For a $t$-dimensional matrix in which all faces of side $s$ in the $x$ direction (for instance) are Hadamard matrices, the codewords can be regarded as being of length $s^{2}$, in which each $s$ coordinates are uncorrelated. This leads to the possibility of much faster decoding. Also by prior choice of the proper direction of the $t$-dimensional matrix, it may be possible to encode more than one message on the same transmission.

\section{Concluding Remarks}

Since writing this paper, the authors have found higher dimensional Hadamard matrices of side $h$ and dimension $>3$ with propriety $(2,2, \cdots, 2)$ for many integers $h \equiv$ $0(\bmod 4)$. higher dimensional orthogonal designs have also been studied further. Details are available from the authors.

\section{REFERENCES}

[1] N. Ahmed and K. R. Rao, Orthogonal Transforms for Digital Signal Processing. Berlin, Heidelberg, and New York: Springer, 1975.

[2] A. V. Geramita and J. Seberry, Orthogonal Designs: Quadratic 
Forms and Hadamard Matrices. New York: Marcel Dekker, 1979

[3] J. Hadamard, "Résolution d'une question rélative aux determinants," Bull. des Sciences Math., vol. 17 pp. 240-246, 1893

[4] H. F. Harmuth, Transmission of Information by Orthogonal Functions, 2nd ed. Berlin, Heidelberg, and New York: Springer, 1972.

[5] M. Harwit and N. J. A. Sloane, Hadamard Transform Optics, New York: Academic, 1979

[6] R. E. A. C. Paley, "On orthogonal matrices," J. Math. Phys., vol. 12, pp. 311-320, 1933.

[7] K. Sawada, "Hadamard matrices of order 100 and 108," Bull. Nagoya Inst. Technol., vol. 29, pp. 147-153, 1977.

[8] P. J. Shlichta, "Three- and four-dimensional Hadamard matrices," Bull. Amer. Phys. Sac., Ser. 11, vol. 16, pp. 825-826, 1971.

[9] -, "Higher dimensional Hadamard matrices," IEEE Trons. In form. Theory, vol. IT-25, no. 5, pp. 566-572, 1979.
[10] J. Seberry Wallis, "On the existence of Hadamard matrices," $J$. Combinatorial Theory, Ser. A, vol. 21, pp. 188-195, 1976.

[11] _ - "Construction of Williamson-type matrices," Linear and Multilinear Algebra, vol, 3, pp. 197-207, 1975.

[12] - - "Hadamard matrices," in Combinatorics: Room squares, sum free sets, Hadamard matrices. Part 4, W. D. Wallis, A. Penfold Street and J. Seberry Wailis, vol. 292, Lecture Notes in Mathematics. Berlin, Heidelberg, and New York: Springer, 1972, pp. 275-489.

[13] R. F. Scott, Theory of Determinants. 2nd Ed., Cambridge, MA: Cambridge Univ. 1904.

[14] J. Williamson, "Hadamard's determinant theorem and the sum of four squares," Duke Math. J., vol. 11, pp. 65-81, 1944.

[15] A. C. Mukhopadhyay, "Infinite classes of Hadamard matrices," $J$. Combinatorial Th., Ser A, vol. 25, pp. 128-141, 1978. 